\section{Patient attitudes to receiving copies of outpatient clinic letters from the ocular oncologist to the referring ophthalmologist and GP}

Y Krishna' and BE Damato 2
${ }^{1}$ Unit of Ophthalmology Department of Medicine University Clinical Departments, Duncan Building, Daulby Street Liverpool, L69 3GA

${ }^{2}$ St. Paul's Eye Unit $8 Z$ link, Royal Liverpool University Hospital, Prescot Street, Liverpool. L7 8XP

Correspondence: Y Krishna E-mail:ykrishna02@ hotmail.com

Received: 23 May 2004 Accepted: 7 September 2004

Published online:

15 October 2004

\begin{abstract}
Aim To investigate patients' views and understanding on receiving a copy of the outpatient clinic letter from the ocular oncologist to the referring ophthalmologist and GP.

Methods Face-to-face interviews were conducted with 52 patients attending outpatient ocular oncology follow-up clinics, in a semistructured format using a qualitative open-ended questionnaire. The clinics are held at Liverpool Ocular Oncology Centre, a tertiary specialist referral centre at St. Paul's Eye Unit in Royal Liverpool University Hospital.

Results Patients' views on receiving a copy of the outpatient letter, their understanding of the letter, and improvements suggested. A total of $58 \%$ of patients had received a letter and $97 \%$ of them said they were glad they had it. Of this group $77 \%$ had shown it to family/ friend. Consistent comments included: 'help with accepting the news;' 'good to be informed and to know what to expect; 'confirmed what was said in the consultation.' Of the patients who had not received a copy of the letter, $64 \%$ replied that they would have liked a copy. A total of $80 \%$ of patients reported that they fully understood the letter. In all, $17 \%$ wanted medical terms to be explained, when asked to suggest improvements.

Conclusions Sending patients copies of the consultant outpatient letter seemed to be highly appreciated and a useful method of information giving regarding diagnosis and management.
\end{abstract}

Eye (2005) 19, 1200-1204. doi:10.1038/sj.eye.6701740; published online 15 October 2004

Keywords: outpatient clinic letters; patient views; information giving

\section{Introduction}

There has been a growing trend towards patient involvement in their own health care, with greater emphasis on communication.

Previous studies have focussed on patients' views and attendance behaviour on receiving a copy of the GP referral letters to outpatient departments (OPD). ${ }^{1-4}$ Jelley et $a l^{4}$ found that patients appreciated receiving a copy of the letter, demonstrated a greater understanding of and furthermore felt involved in the referral process. Conversely, Hamilton et $a l^{3}$ discovered that sending copies of the GP referral letter had no effect on non-attendance rates at OPD. Further down the care process, studies in various hospital settings have shown that sending copies of specialist outpatient letters to patients have positively influenced patient satisfaction with the amount of information given, also enhancing recall of the consultation and improving patient compliance. ${ }^{1,2,5-7}$

However, little research has been undertaken to assess patients' views in receiving copies of the consultant outpatient letter in some of the more stressful areas of hospital care, such as oncology.

The aim of this study was to determine patients' views and understanding on receiving a copy of the consultant letter after attending the Liverpool Ocular Oncology Centre (LOOC). 


\section{Methods}

\section{Setting}

The study was conducted during three consecutive follow-up clinics at LOOC, a tertiary specialist referral centre, at St. Paul's Eye Unit in the Royal Liverpool University Hospital.

Following the initial consultation at the ocular oncology clinic, copies of the outpatient letter were sent to the referring specialist, GP and to the patient. Hence, in order to check whether or not this information was communicated to the patient, a qualitative study was undertaken at follow-up clinic consultations to determine: (1) whether or not a copy of the outpatient letter was received by the patient; (2) if patients understood and their views on the content of the letter; and (3) if they found it useful or not. Patients were briefed on the study, and on obtaining their informed consent were then included into the study and interviewed.

Patients were interviewed on an opportunistic in order to avoid delaying the patients in the follow-up process. Patients were excluded if they were attending for their first consultation with the ocular oncologist, or if they refused to participate.

Of the 58 patients approached, six refused. A total of 52 patients were therefore interviewed. The group comprised 30 females and 22 males, aged between 24 and 87 years (median 61 years). The clinical diagnoses comprised: posterior uveal melanoma (34 patients), suspicious naevus (seven patients), iris melanoma (three patients), choroidal haemangioma (three patients), conjunctival melanoma (two patients), conjunctival lymphoma (one patient), Schwannoma (one patient), and retinal angioma (one patient).

\section{Study design}

Face-to-face interviews were conducted using a semistructured format. A qualitative, open-ended questionnaire was used as an interview guide (please see Appendix A for copy of questionnaire). The interviews took place in a quiet room separate to clinics, with each patient individually and in the presence of any relative/ friend(s) if they wished, and by the same interviewer in order to minimise inter-observer variation. The reliability, content validity, question ambiguity and consistency of the questionnaire, and applicability and feasibility of the study were determined by members of the oncology firm after a small pilot study of five patients.

\section{Data analysis}

Patients' answers to the questionnaire were collected and were analysed in three broad categories: letter received and patients' views; understanding; finally patients' suggestions. Most data were purely qualitative and not amenable to statistical testing. Questions regarding patients' views that were simply answered as 'Yes' or 'No' could be analysed statistically. Categorical data were formally tested using the $\chi^{2}$ test for difference of proportions. Where the $\chi^{2}$ test was invalid, Fisher's exact test was used.

\section{Results}

In all, 52 out of a possible 58 patients took part in the study. The response rate was thus $90 \%$. The answers for each of the categories are given below and summarised in Table 1.

Table 1 Table showing the results for the three categories analysed for patients who had received a copy of the outpatient clinic letter and for patients who had not received a copy of the letter (total, $n=52$ )

\begin{tabular}{|c|c|c|c|c|}
\hline & & $\begin{array}{l}\text { Received } \\
\text { letter (\%) }\end{array}$ & $\begin{array}{l}\text { No letter } \\
\text { received (\%) }\end{array}$ & P-value \\
\hline \multirow{5}{*}{$\begin{array}{l}\text { Letter received and } \\
\text { patients' views }\end{array}$} & Letter received & $30(58)$ & $22(42)$ & 0.33 \\
\hline & Read letter & $30(100)$ & $\mathrm{N} / \mathrm{A}$ & $\mathrm{N} / \mathrm{A}$ \\
\hline & Show letter to or discussed diagnosis with family/friend & $23(77)$ & $21(95)$ & 0.12 \\
\hline & Discuss letter and/or diagnosis with GP & $10(33)$ & $11(50)$ & 0.23 \\
\hline & Prefer to have copy of letter/would have liked copy & $29(97)$ & $14(64)$ & 0.003 \\
\hline \multirow[t]{4}{*}{ Understanding } & Completely & $24(80)$ & $\mathrm{N} / \mathrm{A}$ & $\mathrm{N} / \mathrm{A}$ \\
\hline & Fair extent & $4(13)$ & $\mathrm{N} / \mathrm{A}$ & $\mathrm{N} / \mathrm{A}$ \\
\hline & Little unsure & $2(7)$ & $\mathrm{N} / \mathrm{A}$ & $\mathrm{N} / \mathrm{A}$ \\
\hline & Felt adequate information given & $30(100)$ & $\mathrm{N} / \mathrm{A}$ & $\mathrm{N} / \mathrm{A}$ \\
\hline \multirow[t]{3}{*}{ Suggestions } & None & $23(77)$ & $\mathrm{N} / \mathrm{A}$ & $\mathrm{N} / \mathrm{A}$ \\
\hline & Medical terms explained & $5(17)$ & $\mathrm{N} / \mathrm{A}$ & $\mathrm{N} / \mathrm{A}$ \\
\hline & Other & $2(7)$ & $\mathrm{N} / \mathrm{A}$ & $\mathrm{N} / \mathrm{A}$ \\
\hline
\end{tabular}




\section{Letters received and patients' views}

In all, 30 patients out of the $52(58 \%)$ had received a copy of the outpatient letter explaining their diagnosis and management, whilst $22(42 \%)$ patients had not. This was because the practice of sending copies of letters was only introduced a year earlier.

All patients who received the letter had read the letter themselves and 23 out of $30(77 \%)$ had shown the letter to family or friend. Of the seven patients who had not shown the letter (23\%), four lived alone and three had discussed their diagnosis with family or friend. In all, 10 (33\%) patients had discussed the letter with their GP and found this helpful, 17 felt they already had enough information from follow-up and three were no longer in touch with their GP.

When asked if they preferred the letter to be sent only to the GP, 29 out of 30 (97\%) said 'no' and only one patient was unsure as to why they had received a letter. All who had received the outpatient letter were glad that they had read it and when asked how they felt, consistent comments included: 'know what to expect,' 'help accept news,' 'confirmed in writing what we were told in the consultation,' 'explained and helped understanding,' 'good to be informed... in patient's interest.' One patient felt it was courteous.

Of the 22 patients who had not received correspondence, 14 patients $(64 \%)$ replied that they would have liked to have had a copy, six (27\%) were neutral and two (9\%) said they did not want to know. Patients who had received the letter were significantly more likely to support having such correspondence as compared to the group with no letter $(P=0.003)$. In all, 21 patients $(95 \%)$ had already discussed their diagnosis with family or friend and said they would have shared the letter. When asked about the GP input, 11 (50\%) answered that the GP had been very supportive whereas nine $(41 \%)$ felt they already had enough information. There was no significant difference found between the groups regarding discussing the diagnosis with family/ friend or GP ( $P=0.12$ and 0.23 , respectively).

\section{Understanding}

Of the 30 patients who had a copy of the outpatient letter, $24(80 \%)$ responded that they completely understood the content, four (13\%) understood to a fair extent and two (7\%) were unsure. Medical terminology was the problem in the latter two patients but they still appreciated having a copy of the letter. All patients who had received the letter felt that there was adequate information and that all essentials were covered.

A total of 20 patients $(67 \%)$ answered that they did not feel they needed any further information, whereas 10
(33\%) would have liked further leaflets, for example, providing details on diagnosis and/or actual treatment.

\section{Patients' suggestions}

Out of the 30 patients $23(77 \%)$ felt the letter was fine and could not suggest any improvements. Five (17\%) wanted the medical terms to be explained, but still understood the letter. One $(3 \%)$ patient reported that there was too much detail, such as measurements of the lesion, but recognised that this would be of interest to the referring specialist. One (3\%) patient suggested inclusion of directions to the hospital.

When asked about the adequacy of the questionnaire in assessing their views: 40 of the 52 patients (88\%) replied that the questions seemed valid and that they understood what the questionnaire was aiming to achieve. Three patients $(6 \%)$, said that a long time had elapsed since they had received correspondence from the consultant regarding their diagnosis and management, so it was difficult to remember. Two (4\%) patients found the questions relevant but a little repetitive and one (2\%) was neutral.

\section{Discussion}

Recent studies have demonstrated patient dissatisfaction with information received regarding the referral process and during consultations with hospital specialists. ${ }^{5,8,9}$ Reasons for dissatisfaction include: (1) insufficient information given; (2) poor communication of diagnosis and management; (3) lack of supporting information; and (4) inadequate understanding and recall of information. ${ }^{5,8,9}$ The latter is a particularly common complaint in oncology clinics, as patients informed of their diagnosis of cancer may be too distressed to be able to take in any further details.

Provision of written details to patients regarding their diagnosis and management have been shown to enhance patient understanding and recall of their diagnosis and treatment, and enhance patient involvement in the clinical decision-making. ${ }^{1,2,4-9}$

The aim of this present study was to determine patients' views and understanding on receiving a copy of the consultant outpatient letter, and thus establish the usefulness of this method of information giving.

The feasibility and applicability of the study, and face validity and reliability of the questionnaire were assessed after the pilot study. In addition, most patients reported that the questions seemed valid and that they understood what the questionnaire was aiming to achieve. Although, limitations of the study included: a small sample size; patients taken consecutively at follow-up hence groups were perhaps demographically unmatched; no available 
'gold standard' for assessing patient satisfaction with outpatient clinic letters; and a few patients on long-term follow-up found it difficult to remember back, the present study did highlight salient aspects of doctorpatient communication and information giving.

Of the patients who had received a copy of the clinic letter outlining their diagnosis and management from their initial outpatient assessment, nearly all reported that they preferred having a copy. All of them had read and the majority had shown the letter to family and/or friends. Consistent comments included: help with acceptance of the diagnosis and understanding; confirmed the consultation in writing; felt more informed and involved.

Furthermore, $80 \%$ responded that they completely understood the content, even though the letter had not been modified for them. All patients in this group felt that adequate information was given. The few who were a little unsure of the medical terminology, still appreciated having a copy of the outpatient letter.

Although receiving the letter was generally highly appreciated, some patients expressed that they did not wish to be further reminded of their diagnosis. In consideration of such cases, it may be beneficial to determine whether or not each patient would like further information. However, this may be difficult to achieve.

Similar findings were found by Rutherford $e t a l^{8}$ in an audit of outpatient letters from general renal OPD clinics sent to patients in addition to GPs. In all, $96 \%$ of patients were pleased to receive a copy of the letter and $93 \%$ of them requested copies of subsequent clinic letters. In a separate study, by Damian et $a l^{5}$ patients who had received a letter showed higher total satisfaction scores in information given, with significant improvement in the amount of information recalled, relative to the 'no letter' group. Such trends have been reported particularly in paediatric care where researchers reported increased parental satisfaction and compliance with medical advice, on sending copies of the outpatient letters to parents. ${ }^{1,2,6,7}$

When both groups in this study were asked about their views on the usefulness of different sources of information, most patients replied that they found the audiocassette recording of the initial assessment to be most useful. The remainder found the combination of information sources useful. Previous studies have demonstrated that taping of the consultation to be an effective method of communication and which positively correlates with patient satisfaction. A more recent subsequent survey, undertaken by the unit, of responses of patients with ocular malignancy to taping outpatient consultations reflected the same findings. ${ }^{9}$ In this study, we found patients' ability to understand the letter was enhanced by listening to the audiocassette recording of their initial assessment.
In conclusion, sending patients a copy of the consultant outpatient letter to the referring ophthalmologist and GP increases patient satisfaction. Patients highly appreciated receiving a copy of the clinic letter, felt informed and involved, reported enhanced understanding and recall-especially, when given distressing news in the initial consultation, helped with acceptance, and was a permanent record that patients felt they could refer to. This study demonstrated that sending copies of the consultant oncologist letter to patients, proved to be a useful and valued method of communication with minimal addition to the workload.

\section{Acknowledgements}

I would like to thank Professor Bertil E Damato for the original idea for the study, and I am grateful to him and also Mr Peter Hadden for their help in checking the questionnaire and comments on the study. I would also like to convey my thanks to Mr Steve Taylor, in Department of Mathematical Sciences, University of Liverpool, for his help with statistical analysis of the data.

\section{References}

1 Bailey G, Hyde L, Morton R. Sending a copy of the letter to the general practitioner also to the patients in a child development centre: does it work? Child Care Health Dev 1996; 22(6): 411-419.

2 Cowper DM, Lenton SW. Letter writing to parents following paediatric outpatient consultation: a survey of parent and GP views. Child Care and Health Dev 1996; 22(5): 303-310.

3 Hamilton W, Round A, Sharp D. Effect on hospital attendance rates of giving patients a copy of their referral letter: randomised controlled trial. BMJ 1999; 318(7195): 1392-1395.

4 Jelley D, van Zwanenberg T. Copying general practitioner referral letters to patients: a study of patients' views. $\mathrm{Br} J \mathrm{Gen}$ Pract 2000; 50(457): 657-658.

5 Damian D, Tattersall MH. Letters to patients: improving communication in cancer care. Lancet 1991; 338(8772): 923-925.

6 Eaden JA, Ward B, Smith H, Mayberry JF. Are we telling patients enough? a pilot study to assess patient information needs in a gastroenterology outpatient department. Eur J Gastroenterol Hepatol 1998; 10(1): 63-67.

7 Waterston T, San Lazaro C. Sending parents outpatient letters about their children: parents' and general practitioners' views. Qual Health Care 1994; 3(3): 142-146.

8 Rutherford W, Gabriel R. Audit of outpatient letters. BMJ 1991; 303(6808): 968.

9 Ah-Fat FG, Sharma MC, Damato BE. Taping outpatient consultations: a survey of attitudes and response of adult patients with ocular malignancy. Eye 1998; 12: 789-791. 


\section{Appendix A: patient OPD letter questionnaire}

In order to assess the usefulness of the letter that you were sent following your clinic appointment, please could you complete the following questions. Please circle the answer you feel appropriate and provide any reasons below.

1. Did you receive a copy of the letter that was sent to your GP regarding your diagnosis?

$$
\mathrm{Y} \quad \mathrm{N}
$$

2. Did you read the letter?

Y N

please give reasons

3. Did any member of your family and/or friend read the letter?

$\mathrm{Y} \quad \mathrm{N}$

please give reasons

4. Do you intend to discuss this letter with your GP? Y N

please give reasons

5. Would you have preferred it if we only sent the letter to your GP?

$\mathrm{Y} \quad \mathrm{N}$

please give reasons

6. If you read the letter: $\checkmark$ are you glad that you read the letter?

$\mathrm{Y}$ $\mathrm{N}$

please give reasons and how did you feel

$\checkmark$ did you easily understand the letter? yes, completely, yes, fair extent, little unsure, unsure, not at all.

please give reasons

$\checkmark$ if you found it difficult to understand, do you think it is useful to have a copy of the letter anyway?

$\mathrm{Y} \quad \mathrm{N}$

please give reasons

$\checkmark$ did the letter provide adequate information about the diagnosis and its management?

Y N

please give reasons (what was missing and could perhaps be included)

7. Which of the following did you find most useful? letter, tape (if given one at clinic), patient information booklet, other.

please give reasons

8. Would you have liked to receive further information/ leaflets on your condition and its treatment?

$\mathrm{Y} \quad \mathrm{N}$

9. How could the letter be improved? [e.g. any changes required, what else could it have included] 